\title{
Effect of 4-[(5,6,7,8-Tetrahydro-5,5,8,8-Tetramethyl-2-Naphthalenyl) Carbamoyl]Benzoic Acid (Am80) on Alveolar Regeneration in Adiponectin Deficient-Mice Showing a Chronic Obstructive Pulmonary Disease-Like Pathophysiology
}

\author{
Hitomi Sakai, Michiko Horiguchi, Tomomi Akita, Chihiro Ozawa, Mai Hirokawa, Yuki Oiso, \\ Harumi Kumagai, Yoshito Takeda, Isao Tachibana, Norikazu Maeda, \\ and Chikamasa Yamashita
}

\begin{abstract}
Department of Pharmaceutics and Drug Delivery, Faculty of Pharmaceutical Sciences (H.S., M.Ho., T.A., C.O., M.Hi., O.Y., H.K., C.Y.), and Fusion of Regenerative Medicine with DDS, Research Institute for Science and Technology (M.Ho., C.Y.), Tokyo University of Science, Chiba; Respiratory Medicine, Allergy and Rheumatic Diseases (Y.T., I.T., N.M.), and Department of Metabolism and Atherosclerosis (N.M.), Graduate School of Medicine, Osaka University, Osaka; Department of Medicine, Nissay Hospital, Nippon Life Saiseikai Public Interest Incorporated Foundation, Osaka (I.T.), Japan
\end{abstract}

Received February 1, 2017; accepted March 27, 2017

\begin{abstract}
Chronic obstructive pulmonary disease (COPD) is an intractable pulmonary disease that causes widespread and irreversible alveolar collapse. Although COPD occurs worldwide, only symptomatic therapy is currently available. Our objective is the development of therapeutic agents to eradicate COPD. Therefore, we focused on 4-[(5,6,7,8-tetrahydro-5,5,8,8-tetramethyl2-naphthalenyl) carbamoyl] benzoic acid (Am80), which is a derivative of all-trans retinoic acid. We evaluated the effects of Am80 on alveolar repair in a novel COPD model of adiponectindeficient mice. This mouse model has more symptoms similar to human COPD than the classic elastase-induced emphysema mouse model. Lung volume, computed tomography (CT) values,
\end{abstract}

low-attenuation area ratios, and bone and fat mass were measured by CT. However, the administration of Am80 did not affect these results. To examine the degree of destruction in the alveoli, the mean linear intercept of the alveolar walls was calculated, and assessment of this value confirmed that there was a significant difference between the control $(46.3 \pm 2.3 \mu \mathrm{m})$

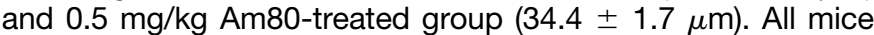
survived the treatment, which lasted for more than 6 months, and we did not observe any abnormalities in autopsies performed at 80 weeks of age. These results suggested that Am80 was effective as a novel therapeutic compound for the treatment of COPD.

\section{Introduction}

Chronic obstructive pulmonary disease (COPD) is the result of various different causes such as smoking which lead to chronic airflow obstruction (Pauwels et al., 2001). COPD had been classified as emphysema or chronic bronchitis until the Global Initiative for Chronic Obstructive Lung Disease established the concept of the disease in 2001. According to the World Health Organization, COPD is expected to become the third largest cause of death in the world by 2030 (WHO, 2016). Currently, no agent is available that can achieve a radical cure of the alveolar destruction caused by this disease (Feldman,

This work was partially supported by the Mochida Memorial Foundation for Medical and Pharmaceutical Research (to M.Ho.), the Hamaguchi Foundation for the Advancement of Biochemistry (to M.Ho.), and the Nagai Memorial Research Scholarship from the Pharmaceutical Society of Japan (to T.A.)

H.S., M.Ho., and T.A. contributed equally to this work.

https://doi.org/10.1124/jpet.117.240515.
2013). As first-line drugs for symptomatic therapy, long-acting muscarinic antagonists, inhaled steroids, or long-acting $\beta 2$ agonists have been used (Karel, 2016; Rodrigo and Neffen, 2017). However, the rate of satisfaction with these agents for COPD is approximately only 40\% (Massaro and Massaro, 1997), which indicates patient dissatisfaction with such treatments. A drug incorporating a new strategy of treatment of COPD is needed.

We have already shown that 4-[(5,6,7,8-tetrahydro-5,5,8,8tetramethyl-2-naphthalenyl) carbamoyl] benzoic acid (Am80) (chemical formula: $\mathrm{C}_{22} \mathrm{H}_{25} \mathrm{NO}_{3}$, CAS number: 94497-51-5) (Fig. 1) can induce the differentiation of human alveolar epithelial progenitor cells into type I and II alveolar epithelial cells (Sakai et al., 2014). We also reported that Am80 showed clear improvement effects on COPD in a mouse model of elastase-induced emphysema (Sakai et al., 2014). This is a pulmonary emphysema model that can be easily produced and has been widely used (Antunes and Rocco, 2011). However,

ABBREVIATIONS: Am80, 4-[(5,6,7,8-tetrahydro-5,5,8,8-tetramethyl-2-naphthalenyl)carbamoyl]benzoic acid; ATRA, all-trans-retinoic acid; COPD, chronic obstructive pulmonary disease; CT, computed tomography; DMSO, dimethylsulfoxide; HU, Hounsfield unit; LAA, low-attenuation area; Lm, mean linear intercept. 


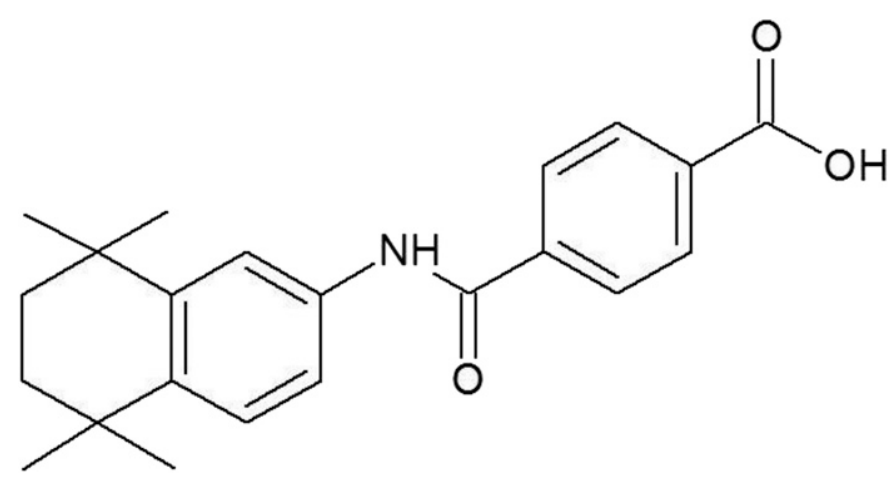

Fig. 1. Chemical structure of Am80.

this model is problematic in that it lacks long-term inflammation, so the reduction of emphysema is also transient. Therefore, we need to focus on a model that better reflects the disease state of human COPD.

It is thought that adiponectin-deficient mice are more suitable as a disease model of COPD than the elastaseinduced emphysema model in regard to these points. However, the effect of Am80 on alveolar regeneration and systemic symptoms has not been evaluated. Adiponectin is a protein secreted from adipocytes (Ouchi et al., 1999). Because of its antiinflammatory effects and improvements in insulin resistance, it has been suggested to contribute to the metabolic syndrome (Welty et al., 2016). Because a decrease in adiponectin also occurs in patients with COPD, adiponectin is strongly suggested to be associated with the pathogenesis of COPD (Couillin et al., 2009), which is still largely unknown. It is thought that the enhancement of apoptosis in endothelial cells caused by the decreased expression of vascular endothelial growth factor-2 and platelet endothelial cell adhesion molecule- 1 is responsible for the development of COPD-like symptoms in adiponectin-deficient mice (Nakanishi et al., 2011). Our present study used adiponectin-deficient mice as a new disease model of COPD and examined the effects of Am80 on alveolar regeneration.

\section{Materials and Methods}

Reagents and Animals. Am80 was a gift from Dr. Koichi Shudo of the Itsu Institute (Tokyo, Japan). Physiologic saline was purchased from Otsuka Pharmaceutical (Tokyo, Japan), and ethanol and dimethylsulfoxide (DMSO) were purchased from Wako Pure Chemical Industries (Osaka, Japan). Isoflurane used for anesthesia in the mice were purchased either from Intervet KK (Osaka, Japan) or Wako Pure Chemical Industries.

The adiponectin-deficient mouse (apM1 Homo) was originally created in the Osaka University School of Medicine. The mice were transferred and have been maintained at CLEA Japan (Tokyo, Japan). The male and female mice were provided by CLEA Japan, and they were bred in our laboratory. All animal care and use procedures were approved by the Tokyo University of Science Ethics Committee (approval numbers Y12058, Y13008, 1542). Male mice were used in the experiments. After evaluating the development of emphysema in the adiponectin-deficient mice, we performed pulmonary administration with the method we describe here in a control group (six mice) and an Am80-treated group (five mice).

The method of pulmonary administration was reported in our earlier studies (Horiguchi et al., 2015, 2016). Pulmonary administration was performed with a gastric tube for oral administration
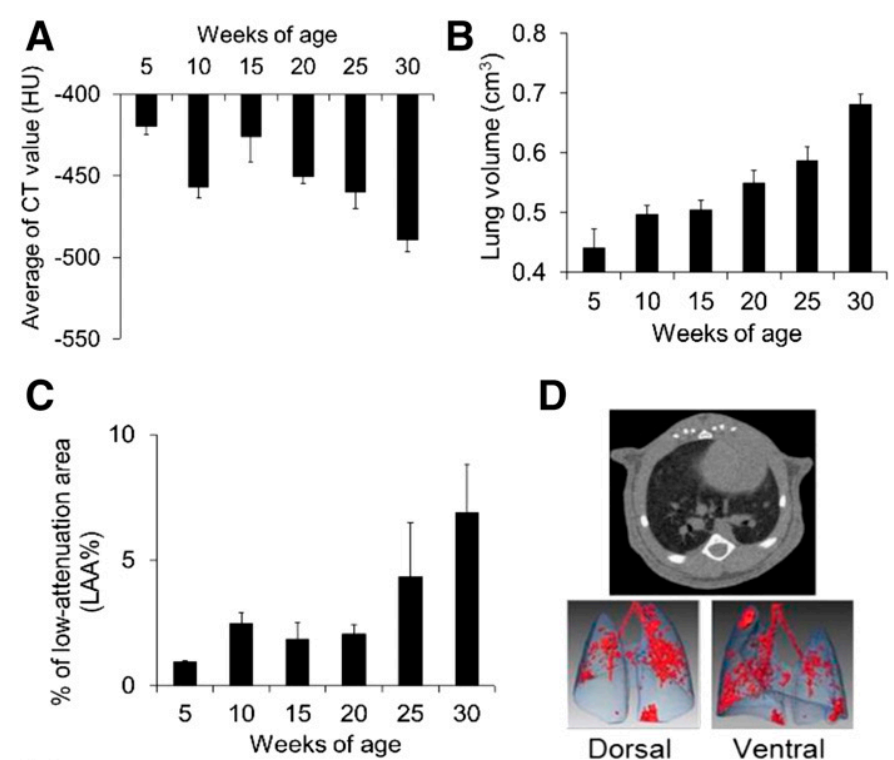

E

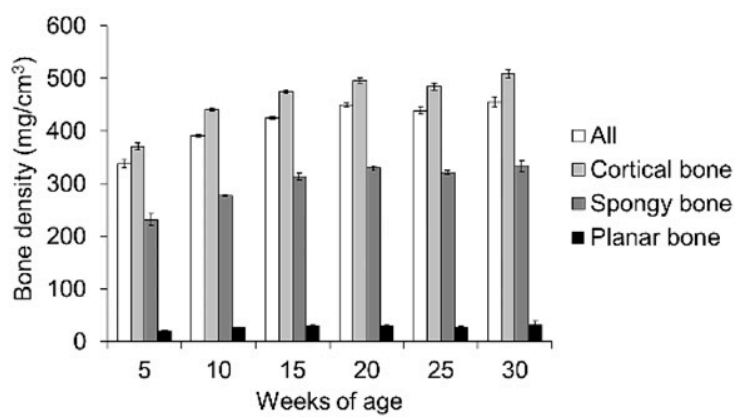

Fig. 2. Micro-CT assessment revealed that adiponectin-deficient mice showed progressive COPD-like phenotype development. (A) The average $\mathrm{CT}$ values, (B) average lung volumes, and (C) average LAA\%. (D) CT image and three-dimensional image by integrating CT images at 30 weeks of age (LAA \% is $1.91 \%$ ). The LAA ( -871 to $-610 \mathrm{HU}$ ) is colored in red, and the whole lung field ( -1000 to $-200 \mathrm{HU}$ ) is colored in blue. (E) Overall, cortical, spongy, and planar bone densities of the chest. Data represent the mean \pm S.E. $(n=5-15)$.

(no. KN-348 for mouse; Natsume Seisakusho, Tokyo, Japan). The gastric tube is equivalent in diameter to the mouse airway. Using the Mouse Intubation Platform-Model MIP (Penn-Century, Wyndmoor, PA) as a mouse retainer for pulmonary administration, the front teeth of the mouse were retained in the retaining position at approximately a $90^{\circ}$ angle to facilitate tracheal access of the tube. The airway was confirmed with the Small Animal LaryngoscopeModel LS-2 (Penn-Century) as a tracheal endoscope for the mouse, the tube was inserted into the airway, and the drug solution was administered in synchronization with the air intake of the mouse.

Evaluation by Computed Tomography. Lung volume, computed tomography (CT) values, and low-attenuation area (LAA) ratios in the mice were measured using an animal experimental X-ray CT apparatus (Latheta LCT-200; Hitachi Aloka Medical, Tokyo, Japan). The machine was automatically calibrated by computer software. Mice were anesthetized by the continuous inhalation of isoflurane. For imaging, the field of view was set at $48 \mathrm{~mm}$, and slice thickness was set at $192 \mu \mathrm{m}$.

The lung volume, average CT value, and their standard deviations were calculated with the following settings. The range of CT values (Hounsfield unit; HU) was set to -1000 to $-200 \mathrm{HU}$ for detecting the region of interest. The radius of noise removal was set at 3 pixels. The LAA was calculated with the range of CT values previously described 
A

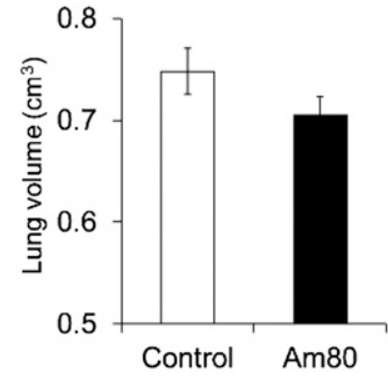

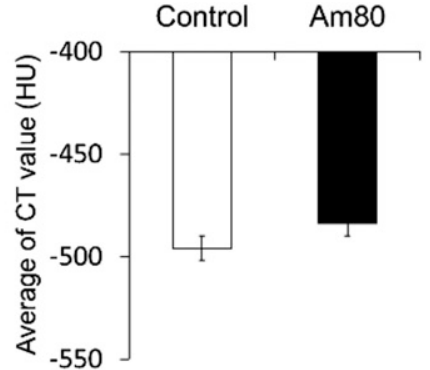

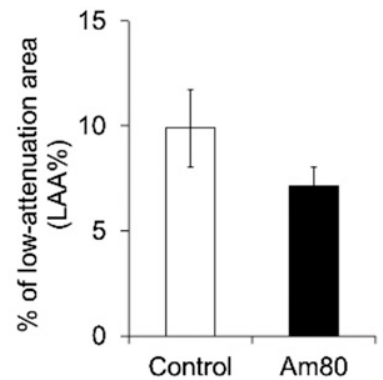

B
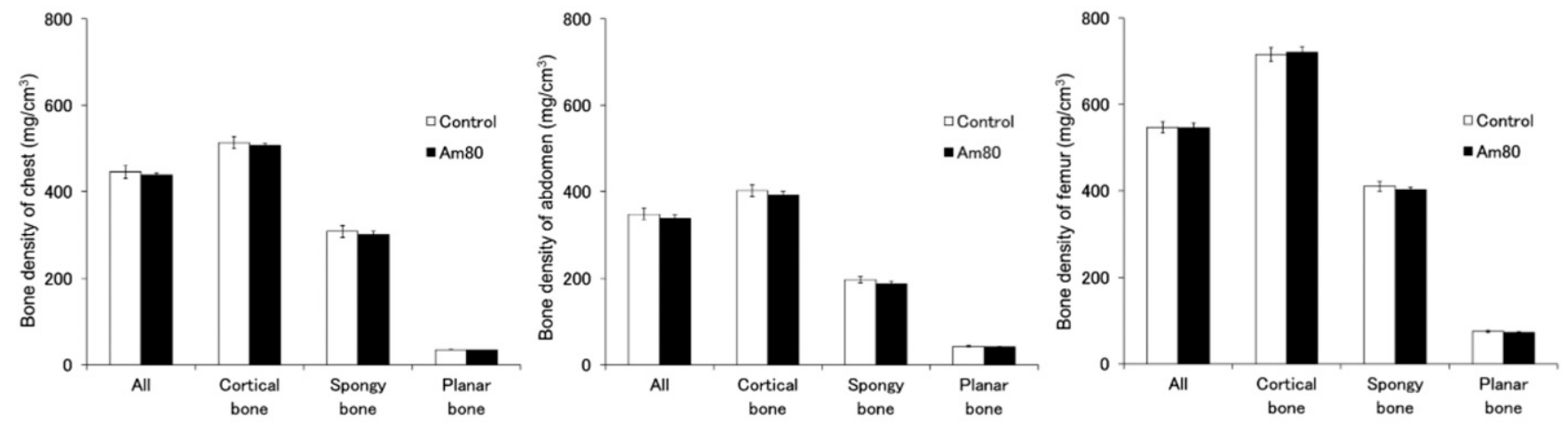

C

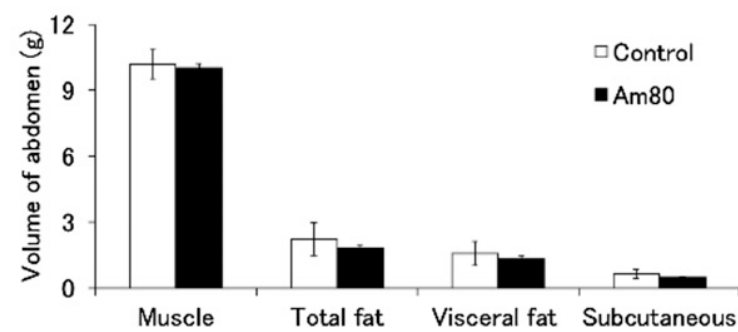

fat

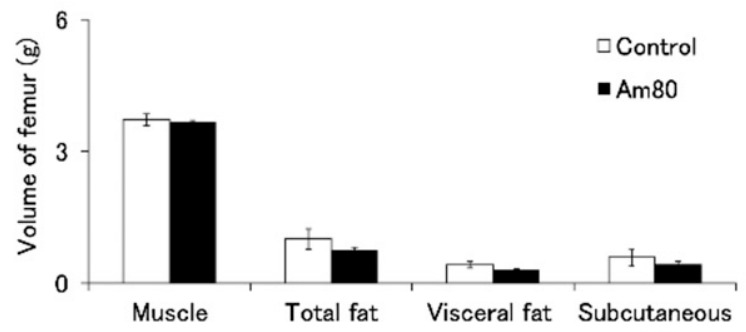

fat

Fig. 3. CT assessment of the repair of lung emphysema in adiponectin-deficient mice at 80 weeks of age. (A) The average CT values, lung volumes, and LAA\%. (B) The overall, cortical, spongy, and planar bone densities of the chest, abdomen, and femur. (C) The volume of muscle and total, visceral, and subcutaneous fat of the abdomen and femur in control (5\% DMSO-treated, $n=6)$ and $0.5 \mathrm{mg} / \mathrm{kg}$ Am80-treated mice $(n=5)$. Data represent the mean \pm S.E.

( -871 to $-610 \mathrm{HU}$ ) (Kobayashi et al., 2013). We used the default setting of the machine to calculate bone and fat mass. Slice thickness was $192 \mu \mathrm{m}$ in the abdomen and $96 \mu \mathrm{m}$ in the femoral region. The scan range of the abdomen was set from the bottom of the ribs to the root of both thighbones.

In addition to the $\mathrm{CT}$ image analysis, lung $\mathrm{CT}$ images were used to create three-dimensional models by Amira, a system for three-dimensional visualization and analysis (Visualization Sciences Group, Burlington, MA). LAA sites in the emphysema area were visualized in red.

Evaluation of Alveolar Wall Distance Using Histochemistry. After the mice were euthanized by an overdose of isoflurane, lung tissue was removed and fixed with $4 \%$ paraformaldehyde/phosphate buffered saline. The fixed lung tissue was embedded with TissueTech OCT compound (Sakura Finetek Japan, Tokyo, Japan). Frozen sections with a thickness of $10 \mu \mathrm{m}$ were prepared using a CM3050S cryostat (Leica Microsystems, Wetzlar, Germany) and then stained with $\mathrm{H} \& \mathrm{E}$. The stained sections were examined at any five points with both $4 \times$ and $20 \times$ magnification using an HS All-in-one Fluorescence Microscope (Keyence Corp., Osaka, Japan).

To examine the degree of destruction in the alveoli, the mean linear intercept $(\mathrm{Lm})$ of the alveolar walls was calculated on the image taken at $20 \times$ magnification: Mean linear intercept of alveolar walls $(\mu \mathrm{m})=$ Number of intersections of the grid lines $\times$ Lattice width $(50 \mu \mathrm{m}) /$ Number of alveoli in the field. Lm was calculated by applying the equation to five different images. The five different images were chosen from each of three sections of lung (upper, middle, and lower parts). We used ImageJ (National Institutes of Health, Bethesda, MD) for this analysis.

Evaluation of Alveolar Repair by Long-Term Administration of Am80 in Adiponectin-Deficient Mice. We used male adiponectin-deficient mice with a C57BL/6J genetic background. We monitored the time course of the degree of emphysema from 5 to $30 \pm$ 1 weeks of age. We justified the use of the adiponectin-deficient mouse model by comparing it to the degree of emphysema in the elastaseinduced emphysema model.

Am80 (0.5 mg/kg) was dissolved in $5 \%$ DMSO/saline solution. We gave $50 \mu \mathrm{l}$ of Am80 solution via pulmonary administration twice a week to the mice. These mice were at $50 \pm 2$ weeks of age and had advanced emphysematous lesions. Control mice received $50 \mu \mathrm{l}$ of $5 \%$ DMSO/saline solution. At 78-82 weeks of age, all mice were euthanized by an overdose of isoflurane. Lung tissue was removed and fixed with $4 \%$ paraformaldehyde/phosphate-buffered saline.

Statistics. For each parameter measured, the values from individual samples were averaged, and the S.E. was calculated. Data were compared using the unpaired $t$ test when only two groups were compared. Dunnett's method was used to evaluate differences between three or more groups. A $5 \%$ probability was considered significant. 


\section{Results}

Time Course for the Development of Emphysema in Adiponectin-Deficient Mice. The elastase-induced emphysema model is a typical COPD disease model that has been used to evaluate the effect of alveolar repair. However, the pathogenesis of emphysema in this model is different from that of human COPD. Moreover, it does not exhibit any systemic symptoms. By contrast, the adiponectin-deficient mouse shows emphysematous symptoms with age, and this mouse also exhibits systemic symptoms, such as increased inflammatory cytokines and decreased bone mass (Nakanishi et al., 2011). Because of these observations, we decided to use this mutant mouse to evaluate the development of emphysema. We evaluated CT values every 5 weeks from 5 to 30 weeks of age.

According to previous reports, in the elastase-induced emphysema mouse model the administration of elastase (4.05 EU) twice a week significantly increased the CT value from $-408 \pm 7 \mathrm{HU} 2$ weeks before injection to $-437 \pm 8 \mathrm{HU}$ 2 days after injection $(P<0.05)$. On the basis of this result, we set the CT value of $-450 \mathrm{HU}$ as representative of the onset of emphysema. At 5 weeks of age, the CT value of the adiponectin-deficient mice was $-420 \pm 4 \mathrm{HU}$, which did not reach the set threshold for emphysema. The $\mathrm{CT}$ value in mice of 20 weeks of age or older dropped to less than $-450 \mathrm{HU}$. Thus, the adiponectin-deficient mice exhibited moderate progression of emphysema along with age (Fig. 2A).

Lung volume was also increased with age (Fig. 2B). As with the CT value, a gradual change in LAA\% was observed, which indicated moderate progression of emphysema with age (Fig. $2, \mathrm{C}$ and $\mathrm{D}$ ). Bone mass of the chest gradually increased up to 20 weeks of age because of growth and became stable without any significant fluctuations (Fig. 2E). Body weight increased from $19 \pm 1 \mathrm{~g}$ at 5 weeks of age to $31 \pm 1 \mathrm{~g}$ at 20 weeks of age. After 20 weeks of age, there was no significant increase in body weight. The LAA\% data are representative of two individual experiments, and the other data are representative of one individual experiment.

Effect of Long-Term Administration of Am80 on Alveolar Repair. From a dose-determination study for Am80, we found that $0.5 \mathrm{mg} / \mathrm{kg}$ is the highest dose that can be administered without mortality and obvious adverse events. We chose as the model animal the adiponectindeficient mouse because it exhibits moderate progression of emphysema and systemic symptoms, such as reduction of bone mass with age. Therefore, we evaluated the effects of the long-term administration of $0.5 \mathrm{mg} / \mathrm{kg}$ of Am80 on alveolar repair by calculating CT or $\mathrm{Lm}$ values in these mice.

We measured the lung volume and CT value in both Am80 and control animals at 80 weeks of age. The lung volume was $0.748 \pm 0.023 \mathrm{~cm}^{3}$ in the control group and $0.705 \pm 0.018 \mathrm{~cm}^{3}$ in the Am80-treated group. The CT value was $-496 \pm 6 \mathrm{HU}$ in the control group and $-484 \pm 6 \mathrm{HU}$ in the Am80-treated group. For LAA\%, the values were $9.90 \pm 1.8 \%$ in the control group and $7.12 \% \pm 0.92 \%$ in the Am80 group (Fig. 3A). Cortical bone, bone surface, total bone, and planar bone densities were not significantly different in the chest, abdomen, and femur between treatments (Fig. 3B).

Total fat amounts in the abdomen, visceral fat mass, and subcutaneous fat mass were also not significantly different between the treatments (Fig. 3C). The percentage of fat in the

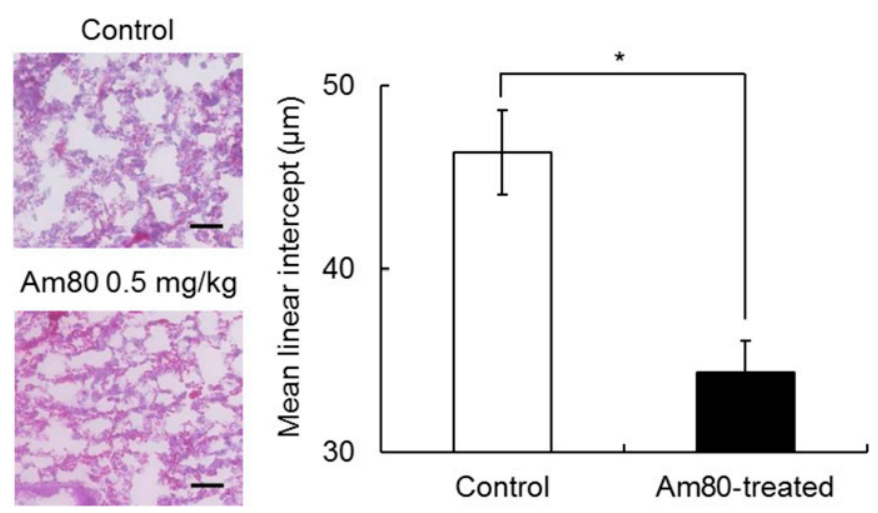

Fig. 4. Lung emphysema repaired with Am80 in adiponectin-deficient mice at 80 weeks of age by intercept assessment. Lung sections and the mean distance between alveolar walls (Lm) from control (5\% DMSOtreated, $n=6)$ and $0.5 \mathrm{mg} / \mathrm{kg}$ Am80-treated mice $(n=5)$ are shown. Sections were stained with H\&E. Scale bar $=50 \mu \mathrm{m}$. Data represent the mean \pm S.E. $* P<0.05$

abdomen was $16.8 \% \pm 4.2 \%$ in the control and $15.2 \% \pm 1.4 \%$ in the Am80-treated group, whereas that in the femur was $20.5 \% \pm 3.6 \%$ in the control group and $16.8 \% \pm 1.4 \%$ in the Am80-treated group.

At the beginning of the treatment at 52 weeks of age, the body weight was $37 \pm 2 \mathrm{~g}$ in the control group and $36 \pm 1 \mathrm{~g}$ in the Am80-treated group. At the end of the treatment at 80 weeks of age, it was $36 \pm 2 \mathrm{~g}$ in the control group and $34 \pm 0.4 \mathrm{~g}$ in the Am80-treated group. Although the body weight was observed to slightly decrease before 80 weeks of age, we did not observe any individual whose body weight decreased to approximately $25 \mathrm{~g}$, as was reported previously (Nakanishi et al., 2011).

The length of $\mathrm{Lm}$ was $46.3 \pm 2.3 \mu \mathrm{m}$ in the control group and $34.4 \pm 1.7 \mu \mathrm{m}$ in the Am80-treated group. Because this difference was statistically significant, we concluded that Am80 has a significant effect on alveolar regeneration (Fig. 4). It should be noted that all mice survived because of the treatment, which lasted for more than 6 months, and we did not observe any abnormalities in the autopsy performed at 80 weeks of age. The LAA\% data are representative of two individual experiments, and the Lm data are representative of three individual experiments. Other data are representative of one individual experiment.

\section{Discussion}

Although COPD occurs worldwide, only symptomatic therapy is currently available. The development of a therapeutic drug to eradicate COPD was the objective of our study. Alltrans-retinoic acid (ATRA) is known to have an effect on alveolar repair in COPD (Massaro and Massaro, 1997). Am80, a derivative of ATRA, is more stable than ATRA. Our present study evaluated the effects of Am80 on alveolar repair in adiponectin-deficient mice.

First, we administered Am80 to adiponectin-deficient mice, which is a novel COPD model. This mouse model has more symptoms similar to human COPD than the classic elastaseinduced emphysema mouse model. It exhibits COPD-related systemic symptoms, such as emphysema, bone loss, and fat mass loss (Nakanishi et al., 2011). The progression of emphysema 
symptoms was observed between 5 and 30 weeks of age (Fig. 2, A-D). This result is similar to that of previous studies.

Although a decrease in body weight and bone mass with age has been reported, we could not reproduce this finding (Fig. $2 \mathrm{E})$. Although the total bone density at 30 weeks of age was $455 \pm 9 \mathrm{mg} / \mathrm{cm}^{3}$, that at 80 weeks of age was $445 \pm 15 \mathrm{mg} / \mathrm{cm}^{3}$ in the control group and $438 \pm 5 \mathrm{mg} / \mathrm{cm}^{3}$ in the Am80-treated group. The difference between 30 and 80 weeks of age was not statistically significant (Fig. 3B). The total bone density in the femur was $546 \pm 13 \mathrm{mg} / \mathrm{cm}^{3}$ in the control group and $546 \pm$ $10 \mathrm{mg} / \mathrm{cm}^{3}$ in the Am80-treated group, which is essentially the same as the data in previous reports.

Our results suggest that the adiponectin-deficient mouse is a useful COPD model because of the high reproducibility of spontaneous emphysema and bone loss. We did not observe any significant effect on systemic symptoms, such as bone or fat mass loss, in theAm80-treated group. However, we found significant improvement in alveolar regeneration as indicated by the Lm value (Fig. 4). In previous reports, correlations between micro-CT and histopathologic findings were observed, but not with complete consistency (Kawakami et al., 2008; Saito and Murase, 2012). We thought that $0.5 \mathrm{mg} / \mathrm{kg}$ Am80 showed mild potency for the repair of collapsed alveoli and that this effect was observed only in the histologic data.

We conducted pulmonary administration of elastase in mice in a previous study. In fact, CT imaging performed after 1 week of elastase administration indicated that the drug solution was delivered to the ends of the lungs (Horiguchi et al., 2015). Using this method of administration, we confirmed a significant improvement in $\mathrm{Lm}$ by Am80 treatment. The results of the measurement of tissue levels of Am80 showed a level in lung tissue of $10.9 \mathrm{ng} / \mathrm{mg}$ and that in blood of $0.447 \mu \mathrm{g} / \mathrm{ml}$, indicating a higher level of Am80 in lung than in blood. In the present study, we found that Am80 improves alveolar regeneration in adiponectin-deficient mice, which is a new COPD mouse model. Taken together from the above results, our findings indicate that Am80 may be a new therapeutic agent for the radical cure of COPD.

\section{Acknowledgments}

The authors thank Dr. Koichi Shudo (Japan Pharmaceutical Information Center) for kindly providing Am80.

\section{Authorship Contributions}

Participated in research design: Sakai, Horiguchi, Akita, Yamashita.
Conducted experiments: Sakai, Hirokawa, Oiso, Kumagai.

Wrote or contributed to the writing of the manuscript: Horiguchi, Akita, Ozawa, Takeda, Tachibana, Maeda, Yamashita.

\section{References}

Antunes MA and Rocco PR (2011) Elastase-induced pulmonary emphysema: insights from experimental models. An Acad Bras Cienc 83:1385-1396.

Couillin I, Vasseur V, Charron S, Gasse P, Tavernier M, Guillet J, Lagente V, Fick L, Jacobs M, Coelho FR, et al. (2009) IL-1R1/MyD88 signaling is critical for elastaseinduced lung inflammation and emphysema. J Immunol 183:8195-8202.

Feldman GJ (2013) Improving the quality of life in patients with chronic obstructive pulmonary disease: focus on indacaterol. Int J Chron Obstruct Pulmon Dis 8:89-96.

Horiguchi M, Hirokawa M, Abe K, Kumagai H, and Yamashita C (2016) Pulmonary administration of 1,25-dihydroxyvitamin D3 to the lungs induces alveolar regeneration in a mouse model of chronic obstructive pulmonary disease. $J$ Control Release 233:191-197.

Horiguchi M, Oiso Y, Sakai H, Motomura T, and Yamashita C (2015) Pulmonary administration of phosphoinositide 3-kinase inhibitor is a curative treatment for chronic obstructive pulmonary disease by alveolar regeneration. J Control Release 213:112-119.

Karel DJ (2016) Respiratory conditions update: chronic obstructive pulmonary disease. FP Essent 448:20-28.

Kawakami M, Matsuo Y, Yoshiura K, Nagase T, and Yamashita N (2008) Sequential and quantitative analysis of a murine model of elastase-induced emphysema. Biol Pharm Bull 31:1434-1438.

Kobayashi S, Fujinawa R, Ota F, Kobayashi S, Angata T, Ueno M, Maeno T, Kitazume S, Yoshida K, Ishii T, et al. (2013) A single dose of lipopolysaccharide into mice with emphysema mimics human chronic obstructive pulmonary disease exacerbation as assessed by micro-computed tomography. Am J Respir Cell Mol Biol 49:971-977.

Massaro GD and Massaro D (1997) Retinoic acid treatment abrogates elastaseinduced pulmonary emphysema in rats. Nat Med 3:675-677.

Nakanishi K, Takeda Y, Tetsumoto S, Iwasaki T, Tsujino K, Kuhara H, Jin Y, Nagatomo I, Kida H, Goya S, et al. (2011) Involvement of endothelial apoptosis underlying chronic obstructive pulmonary disease-like phenotype in adiponectinnull mice: implications for therapy. Am J Respir Crit Care Med 183:1164-1175.

Ouchi N, Kihara S, Arita Y, Maeda K, Kuriyama H, Okamoto Y, Hotta K, Nishida M, Takahashi M, Nakamura T, et al. (1999) Novel modulator for endothelial adhesion molecules: adipocyte-derived plasma protein adiponectin. Circulation 100: 2473-2476.

Pauwels RA, Buist AS, Calverley PM, Jenkins CR, and Hurd SS; GOLD Scientific Committee (2001) Global strategy for the diagnosis, management, and prevention of chronic obstructive pulmonary disease. NHLBI/WHO Global Initiative for Chronic Obstructive Lung Disease (GOLD) Workshop summary. Am J Respir Crit Care Med 163:1256-1276.

Rodrigo GJ and Neffen H (2017) A systematic review with meta-analysis of fluticasone furoate/vilanterol combination for the treatment of stable COPD. Pulm Pharmacol Ther 42:1-6.

Saito S and Murase K (2012) Detection and early phase assessment of radiationinduced lung injury in mice using micro-CT. PLoS One 7:e45960.

Sakai H, Horiguchi M, Ozawa C, Akita T, Hirota K, Shudo K, Terada H, Makino K, Kubo H, and Yamashita C (2014) Pulmonary administration of Am80 regenerates collapsed alveoli. J Control Release 196:154-160.

Welty FK, Alfaddagh A, and Elajami TK (2016) Targeting inflammation in metabolic syndrome. Transl Res 167:257-280.

World Health Organization (WHO) (2016) Chronic obstructive pulmonary disease (COPD). Fact sheet 315, November 2016. WHO Media Centre, http://www.who.int/ mediacentre/factsheets/fs315/en/.

Address correspondence to: Dr. Chikamasa Yamashita, Department of Pharmaceutics and Drug Delivery, Faculty of Pharmaceutical Sciences, Tokyo University of Science, 2641 Yamazaki, Noda, Chiba 278-8510, Japan. E-mail: chikamasa_yamashita@rs.tus.ac.jp 